blood on the stone, and in the test tube contain. ing the solution of known human blood. No precipitate or cloudiness occurred in the test tubes containing the solution of blood from the dog, pig, ox or sheep.

\section{NOTES ON THE PRODUCTION OF THE TEST SERUM IN RABBITS. ${ }^{1}$}

W. F. WHI'NEY, M.D., BOSTON.

As a rule, with moderate care there is little danger of infection of the rabbits by the injection. The abdomen should be shaved and the skin thoroughly scrubbed with strong alcohol. The rabbit is held out straight by an assistant taking his ears in one hand and his hind legs in the other. The skin is pinched up with the left hand and the needle introduced by a steady thrust with the right. The only chance is that the point may not be inserted into the peritoneal cavity, but under the loose skin when liability of an abscess forming seems to be greater. Such has been the experience in two of our cases. After withdrawing the needle the abdomen should be again scrubbed with alcohol.

Any syringe that can hold $10 \mathrm{cc}$. and can be properly sterilized is all that is required. The one we have used is a glass antitoxin syringe with a long, relatively large needle.

The serum which can be most readily procured is hydrocele fluid (in any large hospital several are tapped every week, and each one gives about 200 to $250 \mathrm{cc}$. of fluid). Ascitio and pleuritic fluid are not quite so common, but the amount is so much larger that it can be used for more rabbits.

Serum can be kept for a long time if collected in a sterilized bottle under aseptic precautions, and a little chloroform poured in and allowed to remain at the bottom of the flask. The mouth - oan be plugged with sterilized cotton.

These sera have the advantage over those obtained from blood, that they are perfectly clear and do not have to be separated from the blood clot and corpuscles. Human blood serum is most readily obtained by squeezing a freshly delivered placenta and then allowing the serum to separate by clotting. The centrifuge is often needed to clear the serum of any stray corpuscles. About 6 to $8 \mathrm{cc}$. can be thus obtained from each placenta.

As to the relative advantage of the serum from one source or another, our experiments, as yet, are not conclusive. Krause says that hydrocele fluid does not develop as strong a reaction in the rabbit as the serum obtained directly from the blood. On the other hand, Uhlenhuth says there is great difference in the susceptibility of rabbits.

In our experience in two parallel series, the hydrocele rabbit gave the better reaction in one and the blood serum rabbit in the other. Further

'Read before the Massachusetts Medico-Legal Society, Jan.4, experiments are necessary before this can be regarded as settled.

Is there any indication by which it can be known beforehand whether a rabbit will yield a strong antiserum? The only indication whioh we have on this point is that in both of those which gave the best reaction, the blood did not coagulate so quickly as in those which gave a poorer reaction. Whether this is a condition which is developed by the formation of the antiserum, or whether it preexisted, we have no knowledge. If it pre-existed and is more than a coincidence it might be used in selecting the animals for injection.

In two cases a single large dose $(100 \mathrm{cc}$.) of hydrocele fluid was injected at once, but no reaction was obtained either the next day or at the end of a week. So that in this way at least the process cannot be shortened.

It was thought that the antiserum might possibly be secreted by the kidneys, and the urine of a highly sensitized rabbit was collected for this purpose. But no characteristic reaction was obtained.

While the operations and reactions are so simple that they can be performed by anyone, still they require care and exactness, and considerable previous practice should be obtained before one would be qualified to testify in a capital case.

In every case of death by violence, where murder is suspected, and there is a possibility of a blood stain having to be examined, a strip of filter paper should be soaked in the blood of the individual at the autopsy. This should be sent to the expert who makes the examination of the suspected blood stain in order that any doubt as to this individual's blood giving a serum reaction can be set at rest.

\section{NOTES ON X-LIGHT.}

BY WHLLAM ROLLIN, BOSTON.

VACUUM 'TUBE REGULATORS FOR X-LIGHT TUBES.

The transformer type.-A chemical capable of yielding a vapor on heating, reabsorbing it again on cooling, is placed on a suitable shaped receptable, usually a cylinder of glass one centimetre in diameter, which is connected with the $x$-light tube. A wire is laid from each pole of the generator to a coil of fine wire surrounding the cylinder containing the chemical. The secondary of this coil is a few turns of coarse wire, within or without the cylinder. In either case, the arrangement forms a stepdown transformer, in which the high potential current used to excite the $x$-light tube is transformed into a heating current, the effect of which is to liberate vapor from the chemical. The duration of the heat depends upon the length of time the current passes in the fine wire of the transformer, being determined by the length of the variable spark gap in the circuit containing the fine wire coil. The action is auto. 
matic, as in some of the other regulators $I$ have designed. When the resistance of the tube is higher than that of the regulator circuit, a current passes through the fine wire of the transformer, heating the coarse wire of the primary, liberating the gas, which, going into the tube, lowers its resistance until the current no longer passes through the transformer, until by use the resistance of the tube has risen. Thus the vacuum of the tube is maintained at any desired point.

The high potential bridge regulator.-A similar glass cylinder to the one already described, or any other suitable receptacle, contains, in addition to the chemical, a piece of fine platinum wire whose ends are connected with proper terminals sealed into the glass, serving as attachments to the wires from a source of current of low voltage. When a current passes through this wire it is heated. A suitable current may be obtained from four cells of Mesco dry battery. The circuit composed of the fine wire and the battery contains a minute spark gap which prevents the current from the battery from heating the wire. The high potential current is used as an automatic drawbridge on which the low voltage current can cross, enabling it to heat the wire of the regulator, thus lowering the vacuum. To compel the high voltage current to bridge the gap instead of going through the battery, a choking coil or other suitable device is used. The action of this regulator is also automatic. These regulators are described in a general way, as I wish to claim the principles broadly, to prevent patents being taken, for we already have too many of these on $x$-light apparatus, a considerable number being unjust.

For examples, two are mentioned. In $1896^{1}$ Pupin and others recommended the use of fluorescent screens for diminishing the exposures for photography by $x$-light. Such screens are essential at the present time for taking instantaneous pictures of the organs of the chest, which are of great importance, as they give us permanent, instead of fleeting, images, yet the method has been made the subject of a patent. Another case of importance is that of automatic regulators for $x$-light tubes. These have been in use since 1896, and with powerful apparatus they are often of much importance, yet the patent office has granted patents on these old devices. Our patent laws are in need of revision. No patents should be allowed on any device that has been described in print, and none on any methods or apparatus that may be used in medicine.

SOME CONCLUSIONS FROM EXPERIMIENTS ON GUINEA PIGS WHICH ARE OF IMPORTANCE IN THE TRIATMENT OF DISEASE BY $\mathrm{X}$-IIGH'T.

I have made several series of experiments on guinea pigs with $x$-light. $A$ few of the results have been reported in this journal. In the present note will be given some conclusions from the experiments which are of importance in treating disease by $\mathrm{x}$-light.

1 Electrioity, Feb. 12, 1896.
Rœntgen showed that the visual and photographic intensity of $x$-light varied as the square of the distance. Before $x$-light could be used intelligently as a therapeutic agent it was necessary to learn by experiments on animals whether its effect upon the tissues varied in the same way. My experiments showed it did. The bearing of this observation on the treatment of internal disease is more important than at first appears. It shows that the source of $x$-light should be at a distance, not within a few centimetres, as is the universal custom now when using $x$-light for therapeutic work. Unless the distance between the exterior of the patient and the seat of the internal disease is small relatively to the distance between the nearest surface of the patient and the tube, the healthy skin and superficial tissues will be subjected to a much stronger radiation than the internal diseased organs. This observation points to the necessity of using much more powerful apparatus than is generally employed, because with the source of $x$-light at a distance the strength of the radiation striking the diseased tissue is little; the length of exposure must, therefore, be long.

The next matter is the kind of $x$-light to be employed. General opinion considers the proper radiation for therapeutic purposes to be that from a tube of low resistance, because that is the kind that burns. My experiments on guinea pigs showed that all forms of radiation from vacuum tubes which could be classed as $x$-light could burn, produce abortion and death. Therefore, as wo can produce injurious effects with any form of $x$-light, we should learn to use, without injurious effect, the kind best suited for the purpose. The kind must depend upon the seat of the disease to be treated. For superficial diseases we should use the radiation from a tube of low re. sistance. There are two reasons. This kind of $x$-light is most absorbed by these tissues; there fore we are using the energy to the best advantage while subjecting the underlying healthy tissues to less stress. Second, with any given generator the amount of electrical energy we can convert into $x$-light in a tube of low resistance is far greater than the amount we can convert in to $\mathrm{x}$-light in a tube of high resistance. This is hard to believe, for a high resistance tube appears to be giving a light many times as brilliant, but my experiments in pumping tubes prove that as the exhaustion goes on the amount of electricity which any given generator can send through a tube diminishes. One reason why I have always advocated describing a tube as having a high resistanoe rather than as having a high vacuum has been to keep this fact in mind. Because it has not been recognized, we have gone astray, supposing the light that burnt was that from a low tube, and in consequence thought that this was the light required to produce therapeutic effects. It is a question of quantity as well as quality.

If we wish to treat internal diseases, we should use the radiation from a tube of high resistance, because this light being less absorbed by the su- 
perficial tissues, they are less affected relatively than with a radiation which they alsorl, to a greater extent; also, because this radiation is less absorbed by the superficial tissues more is availa. ble for affecting the internal organs.

Perhaps as important a conclusion as any from the experiments is this: We need powerful apparatus, and experimenters who are working hard to design such apparatus should be encouraged. Their results should not be condemned because the photographic results olotained with them in the hands of a few have been uncertain and less satisfactory than those obtained with old-fashioned coils and static machines. An investigation showed me that the fault was not in new and powerful types of generators, but wilh the tulse makers. I have, therefore, published in the Electrical Review simple directions for pumping $x$-light tubes for powerful apparatus.

ON THE NLED OF AN INSTRUMEN'T FOR MEASURINA:



I have suggested that the greatest need in $x$-light tubes was a means of restoring the pristine brilliancy. Though this difticulty is not completely removed, it is no longer of first importance, because a fairly good solution has been found to be the use of more powerful apparatus of the type shown in Note 112." If a generator has sufficient power we can afford to let this initial brilliancy die out and use the light yielded by an old tube with a proper regulator. At the present time the problem of greatest importance is to find instruments for measuring the intensity of $x$-light. When these instruments are discovered and a standard fixed, I hope the x-light unit will be called a Isenard, after the physicist who first.showed that a vacuum tube yielded a radiation which, though generally invisible to the eyes, would photograph through opaque substances. $\Lambda$ the present time an observer in reporting his results with $x$-light usually considers he has described its quality and intensity sufticiently when he has stated that the tube was "hard" or "soft," has given the spark-length of his coil and the amperage of the current used in the primary. Consequently, the results of one man cannot be compared with those of annther, for the amount and intensity of the light, as well as its quality, can vary greatly, the stated terms being alike. One of the required instruments should quickly show to the eyes the therapeutic power of the x-light, and another, as suggested by Mr. Heinze, should tell its photographic intensity. This need is well seen in using large coils of the type mentioned, because it is possible, with the arrangements of condensers and spark-gaps of these coils, for the light to appear brilliant when we look in the fluoroscope and yet to have little actinic power. Until we get an actinometer, the photographic intensity of $x$-light should be judged by a tungsdate of calcium screen and not by one of platinocyanide of barium. Skilled observers who use these powerful coils are alle to make good photo-

" Electric:al leview, Dou, 2fi, 1900. graphs of the hips (the most difticult joints) with certainty in a reasonable time, as compared with the long exposures required to get inferior results with large static machines. Jut others have condemned powerful apparatus, stating that it was not so good as ordinary static machines or small coils, and have said that the amount of $x$-light did not depend upon the power of the apparatus. Such reasoning is illogical. To get a powerful $x$-light we must use a powerful generator. There is nothing in the nature of $x$-light whereby it differ's in this respect from ordinary light. We do not expect to get 1600 -candle power from a 16 candle power incandescent vacuum lamp. If we want more light of the same kind than we can oltain from the latter, there is no way to get it except by using more current, in a suitable lamp. Until we get $x$-light powerful enough to take instantaneous photographs of the heart and other organs (which could be done if money were provided for experiments) we should not slacken our endeavors to construct and learn to use more powerful apparatus. We are at present only in the dim light of an x-light dawn. Few can see clearly what the day will be. Until we get convenient measuring instruments we should, in attempting to describe the amount and kind of $x$-light used in any case we are reporting, abandon the words hard and soft as applied to a tube, for if we were to shuftle all the words in our vocabulary and take any two at random they would be more suitable than these. Nor is it sufticient to say that the vacuum was equal to so many inches of air, for I have shown that the vacuum is but one factor in determining the quality of the light. Nor will the resistance answer as a standard, for two tubes may have the same resistance and yet the light inay be entirely different, as I have already proved. At present it is necessary to know not only the resistance, lut the size of the terminals, their distance apart, the material of which they are made and the current passing. So simple a cliange as having the anode in one tube a target and in another having the target separate from the anode, will greatly change the character of the light, for the subatoms of the cathode stream move more rapidly toward a target that is an anode, and, consequently, we get more $x$-light in this case, for the heat of impact is higher. ${ }^{8}$ We must also know the kind of break used, the kind of current, as well as its amount, and whether condensers are used in the primary or secondary, their capacity in microfarads or in square centimetres of coated eurface, when they take the form of Leyden jars in the secondary. Yet, with all these data to aid him, an observer cannot at present expect to accurately know the amount and quality of the x-light used in another man's experiment. As this retards progress, it must be overcome.

Tus New Jersey Iegislature, through the efforts of the governor, has appropriated $\$ 50,000$ for a state sanatorium for tuberculosis. 